\title{
Editorial
}

\section{Transesophageal echocardiography in infants and children}

\author{
Norman H. Silverman and Isobel A. Muhiudeen
}

$\mathrm{P}$ erusal of the recent literature concerned with cardiology in the young reveals numerous papers published on the topic of transesophageal echocardiography. ${ }^{1-11}$ It has become increasingly clear that, in adults, transesophageal echocardiography provides important information unobtainable from the transthoracic route. ${ }^{12,13}$ This has not, however, been the case in infants and children, where excellent images obtained through the thoracic cage, suprasternal and subcostal windows are the norm. We must question, therefore, whether the prevailing acceptable standards of precordial echocardiography have been met before we accept the many claims of improved diagnostic information that are made by the proponents of the transesophageal approach. In this respect, it should be noted that several of the papers describing patients with congenital cardiac malformations, which purport to offer substantially new information using the transesophageal technique, have been assembled largely using series consisting almost exclusively of adults, with only few infants and children included. It is difficult to believe that reports of this technique as "the" diagnostic one will provide information not previously obtained in $30-40 \%$ of infants and children examined. ${ }^{8}$ In our experience, it is rare not to obtain the required information by the transthoracic route in any more than $5 \%$ of the children studied. Even then, the information subsequently provided by the transesophageal technique is of a subtle and complimentary nature rather than new and different to that established by transthoracic echocardiography. ${ }^{7}$ In this brief review, therefore, we highlight the present deficiencies but potential unequivocal advantages of the transesophageal approach.

\section{Current deficiencies}

It is evident that, when observing the images obtained from the transesophageal route in children, the images obtained by many investigators performing transthoracic echocardiography are of superior quality. Continuing improvements in design of transducers by miniaturization of probes have produced transesophageal images more recently of substantially better quality, but they still do not match those obtained by the transthoracic technique. Furthermore, the opportunity to provide ancillary examinations in children, such as continuous wave steerable Doppler, has been limited when using the transesophageal approach. In our opinion, therefore, with few exceptions, the diagnostic capability of transthoracic echocardiography will prove to be as satisfactory, if not superior, to the transesophageal approach in infants and children. In part, this is because the development of transesophageal echocardiography has been brought about commercially, by the obvious need and clinical demand of the adult population. Unfortunately, the size of the standard probe used in adults makes it inadvisable for use in children weighing under $15 \mathrm{~kg}$. The development of smaller pediatric probes has been pioneered by Japanese manufacturers, who have been faced with a substantial number of obstacles in miniaturizing probes to the size required for infants and children. These obstacles have necessitated diminishing the number of elements so as to fit into a gastroscope varying in size between five to eight millimeters. Because of these difficulties in design, there have been few centers using transesophageal probes in children which are of comparable manufacturing standards with the existing systems generally employed in adults. Then, because manufacturing resources in the United States of America in particular have been limited, only relatively few centers here have had the opportunity to develop extensive experience with the transesophageal technique in children. ${ }^{3-11}$

As a consequence of these various caveats, the reports published at this time involving children are largely descriptive, and have not focused on the limitations and deficiencies of the technique. Introduction of the alternative longitudinal plane in the transducers manufactured for adults, when available for use in younger children, may provide a substantial improvement in the resolution of images obtained. Despite this, it is still reasonable to expect that, because of the interposition of the trachea and bronchial tree at certain levels between the transducer and the heart, that "blind" spots will 
remain from the transesophageal approach. We remain skeptical, therefore, when investigators claim that the transesophageal portal gives information not obtained by standard transthoracic techniques in one-third of infants and children examined. ${ }^{8}$

\section{Established and potential advantages}

Despite our skepticism concerning its role in routine diagnosis, we accept that, as a monitoring device in the operating room and in the postoperative period, where imaging from other approaches is hazardous, difficult or impossible to achieve, transesophageal echocardiography will come to play an increasingly important role in evaluation of children and infants. Intraoperative echocardiography can be performed either by a transesophageal or direct epicardial approach, and borh techniques have their advantages and disadvantages. ${ }^{7-11}$ The transesophageal approach offers the particular opportunity for continuous monitoring, as well as the major additional benefit of remaining distant from the surgical field. ${ }^{7-11}$

When combined with a comprehensive preoperative evaluation, the role of intraoperative echocardiography should be to monitor specific problems and to note the effect of the operation on both pathology and function. In this respect, we cannot overestimate the potential value of transesophageal monitoring both during and after surgery in providing continuous information on the changes effected during and after the operation. This does highlight the issue of manpower, since note must be taken of the deployment of those providing care if this technique is to be fully utilized. At the same time, perioperative monitoring of patients with ultrasound is likely to improve the standard of care by keeping the physician in contact with the patients. Studies of this kind are also likely to prove of benefit while the postoperative patient remains in intensive care. $^{5}$

There are then other opportunities where the transesophageal approach is particularly valuable, such as when access to the thorax is limited as, for example, when other surgical or thoracic procedures have been performed and the field should not be contaminated. Taken together, the uses of transesophageal echocardiography in infants and children appear largely to be of value in the operating room; in the postoperative period; and for the occasional patient in whom imaging is substandard either because they are as big as adults or because they are obese. New uses are also emerging, such as the detection of thrombus at the anastomotic site in cavopulmonary anastomoses performed with either the Glenn or Fontan procedures. Transesophageal evaluation of transcatheter closure of atrial septal defects is similarly considered to be part of the conventional standard of care, while the technique has shown its unequivocal value in children as in adults in the evaluation of prosthetic atrioventricular valves. ${ }^{12,13}$

\section{Orientation of images}

Orientation of the transesophageal four-chamber images are usually in keeping with the convention generally used by pediatric cardiac sonographers. It is fortunate in this respect that, without inverting the sector fan, the orientation of most of the axial and four chamber views are also in anatomical orientation. With the introduction of longitudinal or alternative imaging planes, the issue is less clear. Recommendations have not made nor been widely accepted. To our minds, an anatomic orientation should be used wherever possible. In those situations where the orientation cannot be anatomic, the image should be orientated in the same way as generally used during the precordial examination. This does give some problems. For example, in the long axis plane, the apex of the fan must be inverted to match standard parasternal long axis images.

\section{Problems with outpatient referral}

The question of the site for performance of outpatient transesophageal echocardiography remains an area not addressed in most publications. Should it be done in a routine echocardiography laboratory? Or should it be performed in a suite equipped for children? Whichever avenue is appropriate, a number of ancillary requisites will be needed. An adequately trained individual capable of assessing the condition of the patient, and providing assessment and suction of the airway, is essential. Regardless of age, the laboratory must be fitted with adequate equipment for suctioning and monitoring, including the capacity for pulse oximetry. It is appropriate, from a practical point, to have one person manipulating the controls of the ultrasound machine and another attending to the patient. The options of sedation versus general anesthesia must also be considered. Many gastroenterologists are satisfied with the use of sedation when conducting transesophageal procedures but we have used general anesthesia in our population of adolescents with heart disease. Anesthesia provides greater control of the airway and permits a longer more detailed examination but is obviously more expensive.

\section{Conclusion}

It is clear that the technique of transesophageal echocardiography as used in infants and children has already 
made rapid strides, and that the number of reports about the use of this technique are increasing. The acceptance and acquisition of the appropriate instrumentation, however, is still required if the transesophageal approach is to be placed in the armamentarium of all those who evaluate congenital heart disease in infants and young children.

University of California, San Francisco

M342A, Box 0214

San Francisco, CA 94143-0214 USA

Tel. (415) 4765887

\section{References}

1. Ungerleider RM, Kisslo JA, Greeley WJ, Van Tright P, Sabiston DC. Intraoperative pre-bypass and post-bypass epicardial color flow imaging in the repair of atrioventricular septal defects. J Thorac Cardiovasc Surg 1989; 98: 90-100.

2. Cyran SE, Kimball TR, Meyer RA, Bailey WW, Lowe E, Balisteri WF, Kaplan S. Efficacy of intraoperative transesophageal echocardiography in children with congenital heart disease. Am J Cardiol 1989; 63: 594-598.

3. Stümper OFW, Elzenga NJ, Hess J, Sutherland GR. Transesophageal echocardiography in children with congenital heart disease: An initial experience. J Am Coll Cardiol 1990; 16: 433-441.

4. Ritter SB. Transesophageal echocardiography in children: A new peephole to the heart. J Am Coll Cardiol 1990; 16: 447450.

5. Musewe NN, Smallhorn JF, Dyck J, Freedom RM. Transesophageal echocardiography in a pediatric population.
Circulation 1989; 80(Suppl II): II-186. [Abstract]

6. Kyo S, Omoto R, Matsumura M, Shah P, Ito H. Intraoperative transesophageal Doppler echocardiography in pediatric congenital heart disease. Circulation 1989; 80(Suppl II): II186. [Abstract]

7. Muhiudeen IA, Roberson DA, Silverman NH, Hass GS, Turley KT, Cahalan MK. Intraoperative echocardiography in infants and children with congenital cardiac shunt lesions: Transesophageal versus epicardial echocardiography. J Am Coll Cardiol 1990; 16: 1687-1695.

8. Ritter SB. Transesophageal real-time echocardiography in infants and children with congenital heart disease. J Am Coll Cardiol 1991; 18: 569-580.

9. Roberson DA, Muhiudeen IA, Silverman NH. Transesophageal echocardiography in pediatrics: Techniques and limitations. Echocardiography 1990; 7: 699-712.

10. Stumper O, Sutherland GR, Geukens R, Roelandt JRTC, Bos E, Hess J. Transesophageal echocardiography in evaluation and management after a Fontan procedure. J Am Coll Cardiol 1991; 17: 1152-1160.

11. Roberson DA, Muhiudeen IA, Silverman NH, Turley KT, Haas GS, Cahalan MK. Transesophageal echocardiography of atrioventricular septal defect. J Am Coll Cardiol 1991; 18 : 537-545.

12. Nellesen U, Schnitrger I, Appleton CP, Masuyama T, Bolger A, Fischell TA, Tye T, Popp RL. Transesophageal twodimensional echocardiography and color Doppler flow imaging in patients with mechanical prosthesis in the mitral valve position. Circulation 1988; 78: 848-855

13. Van der Brink RBA, Visser CA, Basart DCG, Duren DR, de Jong AP, Dunning AJ. Comparison of transthoracic and transesophageal color Doppler fow imaging in patients with mechanical prosthesis in the mitral valve position. Am J Cardiol 1989; 63: 1471-1474. 